\title{
Intestinal malrotation presenting outside the neonatal period
}

\author{
R YANEZ AND L SPITZ \\ Institute of Child Health, University of London, London
}

SUMMARY We report 37 patients ranging in age from 1 month to 14 years treated for intestinal malrotation during a five year period. The main presenting features consisted of intermittent attacks of vomiting (15 patients), failure to thrive (seven), and recurrent colicky abdominal pain (seven). The diagnosis was confirmed by gastrointestinal contrast studies in all but three patients. A standard Ladd's procedure comprised the definitive surgical treatment. We emphasise the poor nutritional state at the time of operation ( $49 \%$ of the cases were on or below the third centile). In contrast with neonatal presentation, volvulus of the midgut occurred in only five patients $(14 \%)$ compared with $68 \%$ in neonates with malrotation. There were two deaths in the series. Ninety four per cent of the remaining patients responded favourably to the operative procedure.

Malrotation should be considered in the differential diagnosis of a wide variety of symptoms and should be treated promptly once the diagnosis has been confirmed.

Most communications on errors of rotation of the intestine concentrate on aspects involving neonates, most of whom present with the clinical features of acute obstruction or volvulus. ${ }^{1-3}$ The true incidence of malrotation in infants and children beyond the neonatal period is difficult to estimate as many of these anomalies remain asymptomatic and escape detection throughout life. Clearly, abnormalities of midgut rotation can cause symptoms in the older child and adult, but its rarity can lead to serious delay in diagnosis, and unfamiliarity with the condition may lead to inappropriate surgery. ${ }^{4}$

We have been impressed by the diversity of symptoms in patients presenting beyond the neonatal period, the difficulties in establishing the diagnosis, and the frequency of adhesion obstruction in the long term follow up after surgical correction, all of which merit further elaboration. We have analysed all patients with malrotation treated over a five year period, concentrating on the clinical features of cases older than 4 weeks at presentation and comparing them with infants presenting during the neonatal period.

\section{Patients and methods}

The series consisted of a retrospective analysis of 37 infants and children older than one month treated for midgut malrotation at The Hospital for Sick Children, London, in the five years from September 1979 to August 1984. There were 19 boys and 18 girls and their ages at onset of symptoms ranged from 1 week to 13 years (mean 19 months). The mean age at operation was 32 months, with a range of 1 month to 14 years (Table 1). Twenty four patients $(65 \%)$ manifested symptoms within the first year of life, and the proportion of symptomatic cases increased to $76 \%$ for the first two years of life. Two thirds of the patients underwent surgical correction before the age of 2 years.

Cases of malrotation secondary to other diseasefor example, diaphragmatic hernia and exom-

Table 1 Age of patients at onset of symptoms and at operation

\begin{tabular}{|c|c|c|c|c|}
\hline \multirow[t]{2}{*}{ Age } & \multicolumn{2}{|c|}{ At onset } & \multicolumn{2}{|c|}{ At operation } \\
\hline & No & $(\%)$ & No & $(\%)$ \\
\hline Neonatal period & 9 & (24) & - & - \\
\hline 1-3 months & 11 & (30) & 6 & $(16)$ \\
\hline 3-6 months & 1 & (3) & 8 & (22) \\
\hline 6-12 months & 3 & (8) & 5 & (13) \\
\hline $12-24$ months & 4 & (11) & 6 & (16) \\
\hline $2-5$ years & 5 & (13) & 6 & (16) \\
\hline$>5$ years & 4 & (11) & 6 & (16) \\
\hline Mean (months) & \multicolumn{2}{|c|}{$19 \cdot 3$} & \multicolumn{2}{|c|}{31.9} \\
\hline
\end{tabular}


phalos-as well as those discovered incidentally during another surgical procedure were excluded. The mean postoperative follow up period was 15 months.

Clinical data. The major presenting symptoms were vomiting (15 patients), failure to thrive, (seven), and abdominal pain (seven). Vomiting occurred either as isolated intermittent bouts (13 patients) or as part of an acute abdominal crisis (two patients). In nine patients the vomitus contained bilious material. The abdominal pain was generally described as recurrent and colicky in nature. Other modes of presentation were diarrhoea (two patients), constipation (two), and gastrointestinal haemorrhage, obstructive jaundice, prenatal ascites (detected on ultrasound scan), and acute intestinal obstruction (one each). Nutritionally, 18 children $(49 \%)$ were found to be on or below the third centile for weight for their respective ages (Table 2). Sixteen patients $(43 \%)$ had a total of 24 associated abnormalities (Table 3), including two with intrinsic duodenal obstruction due to an annular pancreas and one infant with chylous ascites. The infant with obstructive jaundice resulting from compression of the common bile duct secondary to chronic midgut

Table 2 Nutritional stage at operation in the 37 patients using Tanner's curves for age/sex

\begin{tabular}{lcr}
\hline Centile & No & $(\%)$ \\
\hline$\leqslant 3$ rd & 18 & $(49)$ \\
$>3$ rd $<50$ th & 16 & $(43)$ \\
$\geqslant 50$ th & 3 & $(8)$ \\
\hline
\end{tabular}

Table 3 Associated anomalies in 16 patients (43\% of total)

\begin{tabular}{ll}
\hline Anomaly & No \\
\hline Congenital heart disease & 5 \\
Mental retardation & 4 \\
Necrotising enterocolitis & 2 \\
Intrinsic duodenal obstruction (annular pancreas) & 2 \\
Oesophageal atresia & 1 \\
Chylous ascites & 1 \\
Hirschsprung's disease & 1 \\
Chylous cyst & 1 \\
Situs inversus & 1 \\
Cystic duplication of stomach & 1 \\
Preduodenal portal vein & 1 \\
Meckel's diverticulum & 1 \\
Myopathy & 1 \\
Craniosynostosis & 1 \\
Smith-Lemli-Opitz syndrome & 1 \\
\hline Total & 24 \\
\hline
\end{tabular}

volvulus is unique and has been the subject of a separate publication. ${ }^{5}$

Investigations. Contrast studies of the gastrointestinal tract were carried out in all but two infants. These two infants were a 3 month old boy who presented with acute intestinal obstruction and a paucity of bowel gas shadow on plain abdominal radiograph and the 5 month old girl in whom a malrotation was found at laparotomy for obstructive jaundice (see above).

Upper gastrointestinal contrast studies (barium meal and follow through examination) were performed on 38 occasions in 35 patients. A diagnosis of malrotation was made in 31 patients $(89 \%)$, but in three patients repeat studies were required before the diagnosis was established. In two additional cases the diagnosis was finally recognised on contrast enema.

The lower gastrointestinal tract was investigated in 17 patients ( $46 \%$ of the total of 37 patients) either by contrast enemas (10 patients) or delay films following the barium meal examination (seven). These showed an abnormal position of the caecum in 13 patients and a normally placed caecum in two and was inconclusive in the remaining two patients.

In two patients, despite normal results of a barium meal examination, malrotation was found at laparotomy performed for persisting clinical symptoms.

Treatment. The diagnosis of malrotation was confirmed at laparotomy in all 37 patients. In five patients $(14 \%)$ there was a midgut volvulus that required derotation, but resection for necrosis was not necessary in any of these cases. Correction of malrotation was performed by the classical Ladd's operation-that is, sectioning bands crossing the duodenum and duodenojejunal loop, widening the base of the mesentery, and positioning the small bowel on the right and the colon on the left side of the peritoneal cavity.

An incidental appendicectomy was performed in 34 patients $(92 \%)$.

\section{Results}

There were two deaths in the series. A 3 month old girl died on the second postoperative day from an associated complex congenital cardiac anomaly. The second death occurred in a 2 year old girl who developed an adhesion intestinal obstruction three weeks after an uneventful Ladd's procedure. This complication remained unrecognised in the local hospital and the child was eventually transferred one week later in a moribund state.

Adhesion obstruction was the most frequent 
Table 4 Nutritional stage at operation and at long term follow up using Tanner's curves for age/sex

\begin{tabular}{lll}
\hline Centile & $\begin{array}{l}\text { At operation } \\
(n=37)\end{array}$ & $\begin{array}{l}\text { At follow up } \\
(n=35)\end{array}$ \\
\hline$\leqslant 3$ rd & 18 & 10 \\
$>3$ rd $<50$ th & 16 & 12 \\
$\geqslant 50$ th & 3 & 13 \\
\hline
\end{tabular}

postoperative complication, occurring in three patients $(8 \%)$, all within one year of the Ladd's procedure. All three required operations. Wound infection developed in two patients $(5 \%)$.

The effect of surgical correction of the malrotation on subsequent nutritional state is shown in Table 4. All children whose preoperative weights were above the third centile showed increased weight gain postoperatively. In contrast, only half of the children with weights below the third centile thrived postoperatively.

Long term follow up revealed an excellent response to the surgical correction of the malrotation in 27 patients $(77 \%)$, with total resolution of all symptoms. In six patients there was complete resolution of the presenting symptoms but improvement in nutritional state failed to occur. These six cases, together with two additional patients whose presenting symptoms persisted postoperatively, had sufficient other reasons for their failure to respond completely to the Ladd's procedure-for example, severe cerebral palsy, dysmorphism, chronic food allergy, etc.

\section{Discussion}

Malrotation of the midgut occurs when the normal rotational process and fixation of the intestine fails to take place during the 12th week of gestation after return into the peritoneal cavity of the midgut loop from the 'physiologic umbilical hernia' ${ }^{67}$ The true incidence of the condition is unknown, but roughly $50-70 \%$ of cases occur during the first four weeks of life. ${ }^{89}$ This implies that a substantial number of cases present beyond the neonatal period. One quarter of our patients manifested symptoms in the neonatal period and over half were symptomatic before the age of 3 months. Despite this, only $16 \%$ presented for surgical treatment in the first three months of life. Failure to recognise the importance and the intermittent nature of the symptoms was responsible for the inordinate delay in referral of patients for surgery.

A number of clinical features of the patients in our series merit further elaboration. Bilious vomiting should always be viewed seriously and urgent investigations undertaken to establish the cause of the vomiting. The diagnosis of malrotation should be considered in the differential diagnosis of failure to thrive. The problem with recurrent abdominal pain is far more complicated. Roughly $10 \%$ of schoolchildren suffer from this symptom and to investigate every child would be futile and unrewarding as well as causing positive harm to most of these children by increasing the level of anxiety in the patient and the family. The colicky abdominal pain associated with malrotation is excruciating and incapacitating and totally unlike that of the more common type of idiopathic recurrent abdominal pain. ${ }^{10}$ Vomiting and colicky abdominal pain in the absence of abdominal distension does not exclude intestinal obstruction as a cause.

In the absence of volvulus the plain abdominal radiograph is of little diagnostic value. Volvulus may be recognised by the presence of air fluid levels in the stomach and duodenum while the rest of the abdomen is fairly gasless. The absence of a caecal gas shadow or the location of the small intestinal loops predominantly on the right side of the abdomen should raise the suspicion of malrotation.

The upper gastrointestinal contrast examination is the preferred study in patients presenting nonacutely. The signs to be noted on this investigation are:

(1) Incomplete duodenal obstruction particularly involving the third part.

(2) Duodenojejunal flexure to the right of the midline.

(3) The proximal jejunal loops lying abnormally on the right side of the abdomen.

(4) A 'high' caecum on delayed follow through films.

The risk of barium aspiration, one of the major objections to upper gastrointestinal contrast studies in the presence of suspected high obstructions, has been overemphasised and can be avoided by carefully introducing small amounts of contrast into the stomach, if necessary by nasogastric tube. Positioning the patient in the right prone oblique position will encourage gastric emptying. In addition to defining the position of the duodenum, one of the chief advantages of the upper gastrointestinal contrast study is that it excludes an associated intrinsic obstruction in the duodenum. ${ }^{11} 12$

Once the diagnosis of malrotation has been established, prompt surgical correction should be carried out. The risk of volvulus, although reduced in the older patient, is still present and can lead to disastrous consequences. Surgical treatment consists of dividing all abnormal bands, maximal widening of the base of the small bowel mesentery, and positioning the small bowel on the right side of the 
Table 5 Comparison of clinical features of patients with malrotation presenting in with those presenting beyond the neonatal period

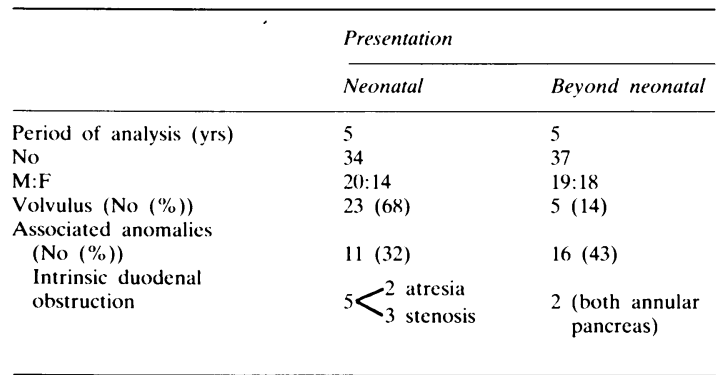

peritoneal cavity and the colon on the left side with the caecum in the left hypochondrium. Routine appendicectomy is generally performed to avoid delay in the diagnosis of appendicitis in the future due to its abnormal position. We have no experience with the operation of 'total correction' or of caecopexy and stabilisation of the mesentery as advocated by Bill. ${ }^{13}$

The major long term complication of the Ladd's operation is adhesion intestinal obstruction, which was noted in three of our patients $(8 \%)$. This increased tendency was also noted in a group of 107 neonates undergoing treatment for malrotation, 15 of whom $(14 \%)$ developed adhesion obstruction. ${ }^{14} 15$

A comparison of the clinical features in neonates presenting with intestinal obstruction and patients presenting beyond the neonatal period during the same five years is shown in Table 5. The features that were of particular interest were the reduced risk of midgut volvulus $(14 \%)$ in patients over 1 month of age compared with cases presenting during the neonatal period and the fairly uncommon occurrence of intrinsic duodenal obstruction.
We would like to thank ODEPLAN-CHILE for sponsoring Dr J R Yanez on his fellowship at the Department of Pacdiatric Surgery, Institute of Child Health, University of London, London.

\section{References}

1 Kiesewetter WB, Smith JW. Malrotation of the midgut in infancy and childhood. Arch Surg 1958;77:483-92.

2 Snyder WH, Chaffin L. Malrotation of the intestine. Surg Clin North Am 1956;36:1479-94.

3 Steward DR, Colodny AL, Daggett WC. Malrotation of the bowel in infancy and children: a 15-year review. Surgery 1976:79:716-20.

${ }^{4}$ Berardi RS. Anomalies of intestinal fixation and position in the adult. Surg Gynecol Obstet 1980;151:113-24.

5 Spitz L, Orr JD, Harries JT. Obstructive jaundice secondary to chronic midgut volvulus. Arch Dis Child 1983;58:383-5.

${ }^{6}$ Dott NM. Anomalies of intestinal rotation. Br J Surg 1923;42:251-86.

7 Synder WH, Chaffin L. Embryology and pathology of the intestinal tract: presentation of 40 cases of malrotation. Ann Surg 1954:140:368-80.

${ }^{8}$ Rickham PP, Lister J, Irving IM. Malrotation and volvulus of the intestine in neonatal surgery. 2nd ed. London: Butterworths, 1978.

9 Filston HC, Kirks DR. Malrotation-the ubiquitous anomaly. J Pediatr Surg 1981:16:614-20

11 Janik JS, Ein SH. Normal intestinal rotation with non-fixation: a cause of chronic abdominal pain. J Pediatr Surgery 1979;14: 670-3.

"Berdon WE, Baker DH, Bull S. Santulli TV. Midgut malrotation and volvulus. Which films are most helpful? Radiology 1970;96:375-83

12 Caffey J. Pediatric $x$ ray diagnosis. 7th ed. Chicago, Illinois: Year Book Medical Publishers, 1978:738-44

13 Bill AH, Grauman D. Rationale and technique for stabilization of the mesentery in cases of non-rotation. J Pediatr Surg 1966;1:127-36.

14 Stauffer UG, Herrmann P. Comparison of late results in patients with corrected intestinal malrotation with and without fixation of the mesentery. J Pediatr Surg 1980;15:9-12.

15 Wilkins B, Spitz L. The incidence of post-operative adhesion obstruction following neonatal laparotomy. $\mathrm{Br} \quad J \quad$ Surg 1986.

Correspondence to Professor L Spitz, Dupartment of Paediatric Surgery, Institute of Child Health, London WC1 N1EH.

Received 14 April 1986 